RESUMEN

\section{La formación de} postgrado de los directores de comunicación españoles

Postgraduate training of Spanish directors of communication

Dra. Kathy Matilla

Facultad de Comunicación y Relaciones Universidad Ramon Llull (URL)

Barcelona (España). kathyms@blanquerna.url.edu

Dra. Thais Miranda

Facultad de Comunicación. Universidade Federal da Bahia (UFBA), Salvador de Bahía (Brasil)

thaisbmiranda@gmail.com

Facultad de Empresay Comunicación.

Universidad de Vic-Central de Cataluña (UVic-CC).

Dra. Andréa Oliveira

Facultad de Turismo. Universidad de Girona (UdG)

Gerona (España). andrea.liveria@udg.edu
Clasificación JEL: $121,123, \mathrm{D} 8, \mathrm{~N} 34$, N36, 052, 054, 057

Palabras clave:

Comunicación

Director de Comuni-

Director de Comuni-

España,

Latinoamérica,

Masters,

Relaciones Públicas

ABSTRACT

JEL Classification: $121,123, \mathrm{D} 8, \mathrm{~N} 34$, N36, 052, 054, 057

Key words:

Matilla, K, Miranda, T., Compte-Pujol, M.y Oliveira, A. (2019)

comunicación españoles

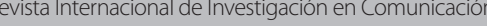

https://doi.org/10.7263/adresic-019-02

Corporate Commu-

nication,

Director of Commu-

nication «dircom»,

Latin American

Masters,

Public Relations,

Spain
El objetivo principal consiste en identificar la formación universitaria de postgrado de los directores de comunicación españoles asociados a Dircom para comprender la vinculación entre los estudios cursados y la orientación y calidad de su praxis. Se ha desarrollado un estudio descriptivo transversal, seguido de un análisis de contenidos. Como limitación al estudio señalar que el tamaño de la muestra no permite cartografiar un mapa generalizado sobre el objeto de estudio, aunque puede considerarse una muestra de oportunidad altamente representativa, de modo que los resultados pueden ser tenidos en cuenta como tendencias. Se observa que la posición se nutre prevalentemente de profesionales formados en postgrados en Comunicación, de tipologías muy atomizadas y especializadas en disciplinas diversas altamente dispersas. Futuras investigaciones son necesarias para comprobar si, en los próximos años, se produce un mayor consenso en los curricula de postgrado de los directores de comunicación españoles. Los resultados obtenidos podrían inferir algunos criterios de contratación de los directores de comunicación españoles en cuanto a formación de postgrado recibida y, así, los resultados de la investigación pueden resultar de interés para orientar a directores generales y de recursos humanos en mejorar el diseño de perfil de conocimientos y competencias requerido para el ejercicio estratégico de la función.

Our main objective is to identify the postgraduate university training of Spanish communication directors associated with Dircom in order to understand the link between the studies they undertake and the orientation and quality of their praxis. To do so, we developed a cross-sectional descriptive study followed by a content analysis. As a limitation to the study, we note that the size of the sample does not allow one to chart a generalized map on the object of study, although it can be considered a highly representative sample of opportunity, and the results can be taken as trends. We observed that the work position of the communication director is predominantly nurtured by professionals trained in postgraduate courses in Communication, of highly atomized typologies and specialized in highly dispersed disciplines. Future research is necessary to verify whether, in the coming years, there is a greater consensus in the postgraduate curricula of Spanish communication directors. The results obtained could infer some recruitment criteria of the Spanish communication directors in terms of postgraduate training received and, thus, the results of the research may be of interest to guide general managers and human resources in improving the profile design of knowledge and skills required for the strategic exercise of the communication director role. 


\section{Introducción}

1.1. Las relaciones públicas y la comunicación corporativa en la clasificación de las áreas de conocimiento

En el «JEL Classification System/EconLit Subject Descriptors» (Journal of Economic Literature) no aparecen introducidas las relaciones públicas ni la comunicación corporativa.

Según Matilla et al. (2014, p. 372), en la «Nomenclatura Internacional de la UNESCO para los campos de Ciencia y Tecnología» tampoco existen como disciplina ni las relaciones públicas ni la comunicación corporativa.

En el listado de áreas de conocimiento adscritas a disciplinas del «Ranking SPI-Scholarly Publishers Indicators-Books in Humanities and Social Sciences», elaborado por el CSIC-Centro Superior de Investigaciones Científicas español, en el área de conocimiento de comunicación únicamente se contemplan la comunicación audiovisual y publicidad (105) y el periodismo (675), sin que aparezca mención alguna a las relaciones públicas ni a la comunicación corporativa.

Todo ello sitúa a los estudios universitarios de grado y de postgrado existentes en España sobre estas dos materias en un limbo de difícil ubicación en el panorama científico y su vinculación con la posición profesional en un lugar de severa indefinición, pese a su creciente implantación en los organigramas de empresas e instituciones españolas e internacionales.

Sin embargo, en la 5a edición del «Ranking I-IGR de Universidades Españolas según Cam-

1 Este estudio se ha llevado a cabo por encargo de Dircom Cataluña, la delegación territorial de la Asociación de Directores de Comunicación Dircom en la comunidad autónoma catalana, a cuya Junta Directiva se agradece la confianza depositada en el equipo de investigación responsable de su diseño y ejecución. pos y Disciplinas Científicas» ${ }^{2}$ aparece la comunicación como disciplina científica del campo «otras ciencias sociales», de forma genérica y sin ningún tipo de desglose.

Que la comunicación corporativa no se considere una disciplina es lógico, porque no lo es: es un área funcional independiente que viene proliferando de forma destacable en los organigramas de empresas y organizaciones españolas e internacionales desde finales de la década de los años 90 del siglo XX.

La Asociación de Directores de Comunicación Dircom postula que el máximo directivo de la gestión de la comunicación debe reportar a dirección general como responsable de diseñar el plan de comunicación alineado con el plan estratégico de negocio y corporativo, ya que el director de comunicación (dircom), integrado en el comité de dirección, debe diseñar y gestionar las estrategias de comunicación desde una perspectiva holística, integrada e integral y asesorar, a nivel staff, a sus homólogos funcionales y a la alta dirección en materia de comunicación (Dircom, 2013).

Por lo tanto, el dircom debe ser un profesional senior y poseer un perfil altamente complejo y con conocimientos multidisciplinares para asesorar a la totalidad de las áreas funcionales de la organización donde presta sus servicios, si bien las técnicas básicas de uso en su departamento son las de las relaciones públicas (Dircom Cataluña, 2013, p. 14), por lo que la formación universitaria especializada en relaciones públicas se

2 Elaborado por los grupos de investigación «EC3-EC3 Metrics spin-off»» y «SCI2S» de la Universidad de Granada (2014) y organizado a través de doce (12) campos y treinta y siete (37) disciplinas cientificas, el «Ranking I-GR»» propone un método de ordenación que sintetiza seis indicadores bibliométricos de producción e impacto científico en un único indicador, el «IFQ2A-Index», empleando como fuente de información las bases de datos reconocidas de Thomson-Reuters: «Web of Science» y «Journal Citations Report», de series temporales amplias (quinquenios, décadas). constituye en fundamental para generar su adecuado perfil de conocimientos («saber») y de competencias («saber hacer»)

En la formación de grado especializada en relaciones públicas en España, que se imparte en los grados mayoritariamente denominados de «Publicidad y Relaciones Públicas» y que es una de las más demandadas (Xifra, 2015, p. 185), se observa una decantación hacia unos curricula con insuficientes créditos en relaciones públicas con respecto a los de publicidad y más orientados a la vertiente técnica -cuando no instrumental-, que estratégica (Moreno, 2004; MorenoFernández et al., 2014; Xifra, 2015), por lo que sus egresados no satisfacen las expectativas de contratación del entry-level del mercado labora de acogida (Xifra, 2015, p. 171)3. Así, son, numerosos los investigadores españoles que han analizado en profundidad los estudios de comunicación en España «que evidencian la falta de adecuación a las exigencias y realidad profesional» (Miquel Segarra et al., 2018: 483), lo que justifica ahondar en el conocimiento sobre la formación universitaria cursada por los dircoms.

Una formación de postgrado de calidad podría suponer una segunda oportunidad para que los graduados españoles especializados en relaciones públicas y/o en comunicación corporativa puedan compensar y superar la deficiente formación de sus primeros estudios universitarios (Matilla et al., 2014, p. 371). Debido a ello, como objetivo principal, nos propusimos identificar en detalle, conocer y reflexionar sobre la

3 Este dato no es patrimonio exclusivo del Estado español, ya que es consistente con la situación estadounidense, donde también son comunes las brechas entre la formación y las necesidades de los empleadores, según describen Soloski (1994); Aldoory y Toth (2000); Hon et al. (2004); o Wright y Turk (2007), manteniendo vigente la idea expresada en el título del libro de Van Ruler (2005) de que «los profesionales son de Venus y los académicos de Marte», por lo que viven de espaldas unos con respecto a otros formación universitaria de postgrado cursada por una muestra de oportunidad altamente representativa de los dircoms españoles y, como objetivo secundario, compararla específicamente con la de sus homólogos latinoamericanos, ya que muchos de ellos han sido formados a nivel de postgrado en España.

\section{El sistema universitario españo}

En el sistema universitario español anterior a Espacio Europeo de Educación Superior (EEES) actualmente en vigor y popularmente conocido como Plan de Bolonia — que es en el que se for maron la mayoría de los sujetos de la muestra analizada-, los másteres contaban con un mayor número de créditos/horas que los postgrados y los programas.

Las versiones «executive» de los másteres españoles poseían -y aún los siguen teniendo- filtros de entrada para sus futuros alumnos, ya que para su acceso/matriculación los estudiantes precisan acreditar su experiencia profesional previa.

Con la integración de España en el EEES junto con otros veintiocho países europeos- $y$ tras una profunda transformación («Real Decreto $56 / 2005$, de 21 de enero» y «Real Decreto 1393/2007, de 29 de octubre»), se crearon los másteres universitarios (también denominados «oficiales»), de entre 60 y 120 créditos europeos (ECTS-European Credits Transfer System), que pueden dar acceso al doctorado tras superar la defensa de un Trabajo Final de Máster y para cuya matrícula se debe contar con una titulación de grado como requisito de entrada.

En el marco del EEES los antiguos postgrados, ahora «oficiales» (mínimo 30 créditos ECTS), han pasado a denominarse «diplomas de especialización». Junto con los másteres universitarios, los actuales diplomas de especialización 
constituyen la única oferta de postgrado «oficial» ofertada en el marco del EEES y, por consiguiente, reconocidos con créditos europeos ECTS

La formación española de postgrado «no oficial» —sin el reconocimiento universitario europeo basado en créditos ECTS-, constituida por másteres, postgrados y programas e impartida por facultades universitarias y/o escuelas de negocio, sigue existiendo y se suelen mantener las nomenclaturas clásicas históricas, si bien se consideran «títulos propios» para diferenciarlos de los «títulos oficiales» con créditos ECTS del EEES y su existencia y vigencia en el tiempo se justifica exclusivamente por su prestigio en el mercado. Suelen ofertarlos las escuelas de negocio, casi siempre pertenecientes o vinculadas a universidades privadas, reservándose los de carácter oficial mayoritariamente a las facultades universitarias

\section{La formación universitaria de los profesionales de la comunicación \\ latinoamericanos}

Los resultados del macro-estudio anual «ECMEuropean Communication Monitor 2015», llevado a cabo con 2.253 directivos de 41 países europeos, revelan que la mayoría $(94,8 \%)$ de los individuos constitutivos de la muestra contaba con un grado en su curriculum académico, un $60,8 \%$ había estudiado un máster y un 7,9\% eran doctores (ECM, 2015, pp. 11 y 14).

El macro-estudio hermano del ECM y de más reciente creación, el «LCM-Latin-American Communication Monitor», está liderado por profesores universitarios de Latinoamérica, España y Estados Unidos, promovido por la European Public Relations Education and Research Association (EUPRERA) y con la Asociación de Directivos de Comunicación Dircom como socio estratégico.
Vinculado al «ECM», el mayor estudio longitudinal sobre la profesión de la gestión de la comunicación y las relaciones públicas de Europa, el «LCM» pretende mejorar el conocimiento de a práctica profesional en Latinoamérica (Argentina, Bolivia, Brasil, Chile, Colombia, Costa Rica, Cuba, Ecuador, El Salvador, Guatemala, Honduras, México, Nicaragua, Panamá, Perú, República Dominicana, Uruguay y Venezuela), evaluando las tendencias para analizar los cambios que se están produciendo en el sector. Pretende visibilizar la función de los comunicadores de habla hispana y portuguesa y empoderar así a esta tipología de profesionales dentro de sus organizaciones, al igual que a las asociaciones del sector dentro de su entorno social.

El «LCM 2014-2015» muestra que las futuras áreas de trabajo más importantes para los 1.774 profesionales de la muestra son las relaciones con la prensa y la publicity (66\%), la comunicación de marketing $(62,7 \%)$ y la comunicación corporativa $(56,1 \%)$.

En cuanto al nivel académico de los participantes del «LCM», el 39,7\% posee un título de graduado, el 51,8\% de máster y el 7,5\% de doctorado.

Es prevalente en los países analizados que se acceda a la profesión mediante estudios superiores que otorgan títulos y diplomas (Argentina 87,3\%; Brasil 85,6\%; Chile 91,0\%; Colombia 68,3\%; Costa Rica 100\%; México 63,2\%; Perú 88,91\%; República Dominicana 72,3\%; Venezuela 93,5\%), si bien cabe señalar la menor coincidencia frente a la consideración de que la comunicación estratégica esté diferenciada de otros campos, como el marketing, la publicidad y el periodismo (Argentina 47,5\%; Brasil 54,7\%; Chile 41,8\%; Colombia 39,6\%; Costa Rica 47,2\%; México 48,5\%; Perú 55,6\%; República Dominicana 38,3\%; Venezuela 41,9\%).
Las variaciones con respecto a que el ejercicio profesional se basa en un cuerpo de conocimiento formal (académico, científico) son notables según cada país (Argentina 48,3\%; Brasi 71,1\%; Chile 41,8\%; Colombia 46,0\%; Costa Rica 80,6\%; México 44,1\%; Perú 66,7\%; Rep. Dominicana 38,3\%; Venezuela 67,7\%) (LCM 2015)

En Colombia, México y Venezuela las relaciones públicas han experimentado una visible expansión; sin embargo, desde la segunda mitad de los años 90 del siglo XX el sector ha sufrido periodos de declive por las incertidumbres económicas y políticas, siendo tres los aspectos que afectan a la profesión en los tres países: la glocalización de la profesión, la inestabilidad económica y las desigualdades sociales (Ibídem).

En Colombia, los profesionales de relaciones públicas se quejan de que los periodistas están inundando la profesión y la mayor parte de los líderes empresariales no comprenden el valor de un proceso sistemático de comunicación como una estrategia fundamental empresarial (Moreno et al., 2006, p. n.e.)

En México, debido a la muy reciente especialización en estudios de relaciones públicas y comunicación corporativa, ninguno de los participantes que ocupan las posiciones más elevada en sus departamentos tenían un título en relaciones públicas. No obstante, la mayor parte habían obtenido una licenciatura en comunicación social (Ibídem)

En Venezuela la situación es similar, ya que la mayor parte de los entrevistados eran licenciados en comunicación social.

Todos los participantes son conscientes de la necesidad de una educación apropiada para ejercer la profesión desde una visión estratégica y, asî, en estos tres países, las primeras amenazas de la profesión provienen de la falta de conocimiento de las verdaderas funciones de relaciones públicas (Ibidem)

«El crecimiento y la especialización de las relaciones públicas se han acelerado en todo el mundo y particularmente en democracias y economías emergentes y en transición [...] afectando y transformando el sector doméstico de comunicación estratégica y relaciones públicas» (Ibídem). Sin embargo, en México, «no se ha extendido una idea clara y correcta de lo que son las relaciones públicas. La actividad de relaciones públicas se confunde todavía con la actividad de organización de eventos» (Moreno y Molleda, 2006, p. n.e.)

En otros estudios sobre Chile se observa que se mantiene al profesional de las relaciones públicas en su dimensión más técnica, en detrimento de la estratégica (Ordeix y Rom, 2014, p. 51)

Por el contrario, la comunicación organizacional en Brasil es un campo institucionalizado gracias a la existencia de programas de postgrado en comunicación, muy consolidados, aunque sigue existiendo incomprensión entre los profesionales de la comunicación acerca de lo que supone su gestión en un contexto organizacional (Oliveira, 2014, pp. 165-166)

\section{La formación universitaria de los profesionales de la comunicación \\ españoles}

En el curso académico 2013-2014 los másteres universitarios (oficiales) españoles especializa dos en relaciones públicas y/o en comunicación corporativa adaptados al EEES se habían multiplicado por seis con respecto al curso 20082009 (Matilla et al., 2014, p. 376)

En 2012-2013 eran 135 los títulos de máster universitario especializados en Comunicación, ofertados por 48 universidades públicas y privadas españolas y que «se han implantado con rapi- 
dez y, en ocasiones, con precipitación e improvisación. Ello ha conllevado que superen en número a los grados» (Castillo-Esparcia et al., 2013, p. 100) La oferta formativa mayoritaria en dicho periodo era de 32 titulaciones específicas de Relaciones Públicas (24\%), 24 (18\%) de Audiovisual; 19 14\%) de Periodismo; 16 (12\%) de Comunicación Social; 12 (9\%) de Comunicación; y 10 7\%) de Publicidad. Por aquél entonces, ninguno de ellos contemplaba el término «Comunicación Corporativa» en su denominación.

García y Gómez (2012a y 2012b) revelaron que en 2011, en España, existían 55 másteres oficiales especializados en Comunicación y Almansa y Athaydes (2012) identificaron que en el curso 2008-2009 los másteres oficiales en relaciones públicas ascendían a 26 , dato contradictorio con el aportado por Hernández et al. (2009), quienes, en el mismo curso, situaban en 25 el número total de másteres en relaciones públicas, de los cuales 21 eran másteres propios (no oficiales) y únicamente 4 eran universitarios.

Así, pues, la integración de España en el EEES ha traído consigo una vasta oferta de formación de postgrado especializada en comunicación y específicamente en relaciones públicas (y posteriormente en comunicación corporativa), tanto oficial como propia que, cuantitativamente, proporciona un amplio abanico de posibilidades formativas para quienes deseen especializarse en dichas materias

En España, la asociación Dircom (2005, $2010,2015 b, 2018$ ) viene proporcionando información, cada cinco años, sobre la titulación de los dircoms obtenida de encuestas realizadas a los profesionales de unas 1.000 grandes empresas españolas, con los resultados siguientes:

- 2005: licenciatura/doctorado en Periodismo 33,5\%; licenciatura/doctorado en Empresa- riales 9,5\%; licenciatura/doctorado en Publicidad y Relaciones Públicas 5,6\%; máster $19,6 \%(n=204)$

- 2010: licenciatura en Periodismo 35,3\%; licenciatura en Empresariales 10,01\%; licenciatura en Publicidad y Relaciones Públicas 5,3\%; máster/doctorado 32,6\% ( $\mathrm{n}=265)$;

- 2015: licenciatura en Periodismo 49,35\%; licenciatura en Empresariales n.e.; máster/doctorado n.e. $(\mathrm{n}=213)$

Datos facilitados en 2018 por Dircom revelan que la licenciatura en Periodismo de sus asociados alcanza el 55,6\%; la licenciatura en Publicidad y Relaciones Públicas 17,6\%; y la formación de postgrado el 55,8\% (especializada en Comunicación 47,5\%) (Dircom, 2018).

Se comprueba que la escasa profesionalización en los niveles superiores y medios de la praxis de las relaciones públicas y de la comunicación corporativa está vinculada a la formación previa recibida, destacando que la mayoría de los profesionales españoles han estudiado Periodismo o provienen de otras disciplinas (García, 2013: 223). Así, las funciones del dircom se ven condicionadas por la procedencia académica y profesional desde la que se accede al cargo, mayoritariamente relacionada con el ejercicio del periodismo que, en 2014, era del 57\% entre los dircoms españoles, frente a solo un $12,68 \%$ proeniente de grados de Publicidad y Relaciones Públicas, así como de otras formaciones (6.57\% Ciencias Económicas y Empresariales; 6,1\% Derecho y Ciencias Jurídicas) (prNoticias, 2014a).

Para ADECEC (2008), la asociación española que agrupa a las mayores agencias de relaciones públicas y comunicación del país, la principal actividad de los profesionales españoles está vinculada a la relación con los media y la publicity (95,2\%), opinión compartida por los lectores de
prNoticias.com (2014b), para quienes esta actividad es también mayoritaria (83\%). La prevalente vinculación de la profesión con el media relations en España resulta consistente con la situación norteamericana descrita por Sha (2011) mientras que, en paralelo, el término «relaciones públicas» se ha desacreditado, tanto en España como en Europa, en beneficio de otras denominaciones como comunicación estratégica o comunicación corporativa (Moreno et al., 2012).

En este confuso contexto los directores de recursos humanos españoles no disponen de una matriz de comportamiento compartida con la que poder racionalizar sus actitudes y actuaciones ante la propia formación, situación esta que puede desvirtuar el comportamiento óptimo que la empresa debería adoptar en lo que respecta a las políticas de formación continua de sus recursos directivos (Araujo de la Mata et al. 2006, p. 84) y, por lo tanto, la política de formación y desarrollo del personal directivo desempeña un papel fundamental como medio para potenciar las capacidades específicas de la empresa y sustentar buena parte de las ventaja competitivas de la organización (Araujo de la Mata et al., 2006, p. 86)

Del mismo modo, no tan solo no discriminan entre postgraduados especializados en Periodismo, Publicidad, Relaciones Públicas, Administración de Empresas, Derecho o Económicas en a contratación de los empleados del área funcional, incluidos sus máximos directivos, sino que se perpetúa la orientación del departamento como gabinete de prensa interno como actividad mayoritaria de la función profesional de la gestión comunicacional, con el agravante de que dichos empleados, con severos déficits formativos acumulados durante los últimos cuarenta años, con el tiempo pasan a contratar a nuevo egresados junior para su departamento, siguien- do los mismos criterios limitativos (Sáez et al. 2015, p. 266)

El análisis de la oferta universitaria en general y de la formación de postgrado en particular en diversas disciplinas es un tema frecuente en el ámbito académico (ej: Rivera et al., 2003; Landeta et al., 2015), considerándose pertinente y adecuado señalar las posibles deficiencias formativas que puedan identificarse en la oferta de sus programas (ej.: Marzo Navarro et al., 2006), y comprobándose que, en este sentido, anteriores estudios han analizado las características e incluso la efectividad de la formación directiva y evidencian la escasa literatura existente en torno a esta cuestión y el desconocimiento por parte de las empresas de los efectos que estos programas tienen sobre sus resultados (Araujo de la Mata et al. 2006, p. 84)

\section{Objetivos}

El propósito principal de este artículo es identificar pormenorizadamente, examinar y comprender la formación de postgrado de los directores de comunicación españoles asociados a Dircom y obtener un mapa de referencia par entender la vinculación existente entre los estudios y la práctica, ya que se sabe que estos profesionales construyen sus carreras con conocimientos académicos (ECM, 2012, p. 77).

Radiografiar los estudios universitarios cursados por los dircoms españoles reviste especia interés ya que diversas investigaciones han puesto de manifiesto la brecha existente entre la formación superior que se imparte en España y las necesidades del mercado laboral (Miquel Segarra et al., 2018: 480).

Cada cinco años la asociación Dircom publica los resultados de la titulación universitaria de los dircoms españoles obtenida de encuestas realizadas a los profesionales de unas 1.000 grandes 
empresas del Estado español (Dircom: 2005, 2010, 2015b), si bien especificando únicamente el porcentaje de ellos que cuentan con formación previa de licenciatura/grado, máster, postgrado y/o doctorado. El valor de esta investigación se centra en que por primera vez se aporta información detallada sobre las tipologías específicas de las titulaciones que complementan las licenciaturas.

Para contextualizar internacionalmente este trabajo, un objetivo secundario se centra en comparar los resultados de la investigación española con los de otros países/regiones, sobre todo de Latinoamérica, ya que casi la mitad $(45,6 \%)$ de los estudiantes extranjeros de los másteres oficiales españoles proceden de América Latina y del Caribe (curso académico 20092010 según Castillo et al., 2013, p. 101), porcentaje que ha alcanzado el 54,8\% en el curso 2014-2015 (Ministerio de Educación, Cultura y Deporte, 2016, p. 71).

La utilidad de esta investigación podría residir en que de los resultados obtenidos se podrían inferir algunos criterios de contratación más ajustados de los directores de comunicación españoles en cuanto a formación de postgrado recibida y, así, pueden resultar de interés para orientar a directores generales y de recursos humanos en mejorar el diseño del perfil de conocimientos y competencias adquiridos en la formación de segundo ciclo universitario requerido para el ejercicio estratégico de la función, de un lado, y diseñar los planes de formación continua ofertados a este tipo de profesionales de forma complementaria.

\section{Metodología}

El objetivo principal de la investigación es identificar la formación de postgrado de los dircoms socios de la asociación española Dircom como primera etapa de futuras nuevas investigaciones que contemplen nuevos planteamientos en preguntas de investigación y nuevas hipótesis que permitan mayor profundización en cuanto a las consecuencias que el perfil formativo identificado en esa muestra de oportunidad puedan tener en la idoneidad y calidad de la praxis profesional de la función comunicativa objeto de estudio.

Se realizó un estudio descriptivo transversal de los datos sobre la formación universitaria de postgrado de los socios de Dircom consignados en el directorio «Quién es quién en Dircom 2015» (Dircom 2015a), que se trasladaron a una plantilla de análisis ad hoc que incluía tres variables: (a) estudios tipo máster; (b) estudios tipo posgrado; y (c) estudios tipo programa que, posteriormente, fueron analizados mediante análisis de contenido (Krippendorff, 2004). Aunque existen ediciones posteriores de dicho anuario (2016 y 2017) se eligió el directorio de la edición anual del ejercicio 2015 para poder cotejar los resultados obtenidos con los de otras investigaciones existentes, identificadas en la lieratura consultada, correspondientes a ese mismo periodo y, con ello, permitir la realización de análisis comparativos en condiciones temporales óptimas

La asociación Dircom, en noviembre de 2015, acogía a 951 asociados (10.11.2015) en su base de datos, siendo buena parte de ellos directores de comunicación de las principales organizaciones españolas (en número de empleados y en facturación), cuyos curricula vitae se recogen en la publicación anual «Quién es quién en Dircom» (Dircom 2015a). Según fuentes consultadas de la propia asociación, unas semanas antes, en el momento de entrar en máquinas el directorio, el número de asociados ascendía a 943 individuos.
Como no todos los socios aceptan que sus datos aparezcan consignados en dicha publicación de los 943 individuos registrados como socios se publicaron los curricula de algo menos de la mitad, en concreto de 435 asociados (46,13\%, n=943), es decir, de casi la mitad del total de los socios censados. En este estudio se contemplo únicamente la muestra final de los 274 dircoms con sus curricula publicados, eliminando el resto de las categorías genéricas de socios: consultores; profesores; conferenciantes, socios de conocimiento y «otros», garantizando, así, la homogeneidad de la muestra.

Se eligió a la asociación Dircom por ser la única del Estado español que acoge a los dircom que, en tanto que tales, desean darse de alta en la organización, permitiendo, además, segmentarlos y diferenciarlos cuantitativamente de otras tipologías de asociados, lo cual convierte a la muestra objeto de estudio en la fuente de información más adecuada y de mayor calidad identificada en el territorio español, pese a la limitación impuesta por la publicación libremente accesible -el directorio anual de socios- que, como ya se ha dicho, únicamente proporciona información del 46,13\% del total de sus asociados.

La cuantificación de la muestra definitiva de los dircoms analizados (274) está en línea con la de los estudios similares realizados quinquenal mente por Dircom (204 en 2005; 265 en 2010 213 en 2015), llegando incluso a ser superior otros estudios realizados con este mismo colectivo (190 en 2014, en el estudio de Miquel Segarra, 2016). La representatividad de la muestra es, pues, limitada ya que se trata de una muestra no probabilística de conveniencia.

El trabajo de campo se realizó por duplicado, de manera consecutiva, y mediante la intervención participante de dos parejas de investigado- res trabajando en paralelo para verificar los datos obtenidos.

\subsection{Preguntas de investigación e Hipótesis}

A partir de la literatura consultada se plantearon cinco (5) hipótesis, vinculadas a tres (3) preguntas de investigación previas, basadas a su vez en tres variables: (a) formación de máster y/o master executive; (b) formación de posgrado; y (c) formación de programa, programa executive y/o programa superior

PI1 ¿Cuál es la formación de máster y/o master executive de los dircoms españoles objeto de estudio?

PI2 ¿Cuál es la formación de posgrado de los dircoms españoles objeto de estudio?

PI ¿Cuál es la formación de programa, programa executive y/o programa superior de los dircoms españoles objeto de estudio?

Hl En las tres tipologías de formación identificadas es mayoritaria la genérica en Comunicación o vinculada con ésta.

H2 En las tres tipologías de formación identificadas es mayoritaria la específica en Relaciones Públicas.

H3 En las tres tipologías de formación identificadas es mayoritaria la específica en Periodismo

H4 En las tres tipologías de formación identificadas es mayoritaria la específica en Marketing

H5 En las tres tipologías de formación identificadas es mayoritaria la específica en Management.

\section{Resultados}

Los estudios de postgrado cursados por los 274 dircoms de la muestra de análisis fueron los siguientes (ver Tabla 1) 


\begin{tabular}{|c|c|c|}
\hline $\begin{array}{l}\text { Master y Master Executive } \\
\qquad \mathrm{N}=243\end{array}$ & $\mathrm{n}$ & $\%$ \\
\hline Master Executive in Business Administration (MBA) & 44 & 18,11 \\
\hline Máster en Dirección Comercial y de Marketing & 10 & 4,12 \\
\hline Máster en Periodismo & 8 & 3,29 \\
\hline Máster en Comunicación Empresarial & 6 & 2,47 \\
\hline Máster en Dirección de Comunicación & 4 & 1,65 \\
\hline Máster en Comunicación Corporativa & 4 & 1,65 \\
\hline Master Comunicación Corporativa y Publicitaria & 4 & 1,65 \\
\hline Máster en Relaciones Internacionales & 4 & 1,65 \\
\hline Máster en Comunicación & 3 & 1,23 \\
\hline Máster en Marketing & 3 & 1,23 \\
\hline Máster en Marketing y Gestión Comercial & 3 & 1,23 \\
\hline Máster en Comunicación Institucional y Política & 3 & 1,23 \\
\hline Máster en Nuevas Tendencias y Procesos de Innovación en Comunicación & 3 & 1,23 \\
\hline Máster en Dirección Estratégica de la Comunicación & 2 & 0,82 \\
\hline Máster en Comunicación Corporativa e Institucional Digital Web 2.0 & 2 & 0,82 \\
\hline Máster en Comunicación Corporativa Digital 2.0 & 2 & 0,82 \\
\hline Máster en Dirección de Empresas & 2 & 0,82 \\
\hline Máster en Protocolo & 2 & 0,82 \\
\hline Máster en RSC & 2 & 0,82 \\
\hline Máster en Periodismo Audiovisual & 2 & 0,82 \\
\hline Master in Business Communication & 2 & 0,82 \\
\hline Máster en Información Económica & 2 & 0,82 \\
\hline Máster en Comunicación Política y Corporativa & 2 & 0,82 \\
\hline
\end{tabular}

\begin{tabular}{|c|c|c|}
\hline Máster en Comunicación Científica & 2 & 0,82 \\
\hline Máster en Marketing y Comunicación & 2 & 0,82 \\
\hline Máster en Dirección de Marketing y Comercial & 2 & 0,82 \\
\hline Máster en Dirección de Comunicación y Publicidad & 2 & 0,82 \\
\hline Máster en Publicidad y Comunicación Empresarial & 2 & 0,82 \\
\hline Master in Internet Business & 2 & 0,82 \\
\hline Máster en Dirección Económico-Financiera & 2 & 0,82 \\
\hline Máster en Unión Europea & 2 & 0,82 \\
\hline Máster en Comunicación y Relaciones Públicas & 1 & 0,41 \\
\hline M.A. in Public Relations Management & 1 & 0,41 \\
\hline Máster en Relaciones Públicas Estratégicas, Empresariales e Institucionales & 1 & 0,41 \\
\hline Máster en Comunicación Empresarial, Institucional y Política & 1 & 0,41 \\
\hline Máster en Dirección de Comunicación Institucional y Empresarial & 1 & 0,41 \\
\hline Máster en Comunicación Estratégica e Innovación en Comunicación & 1 & 0,41 \\
\hline Máster de Comunicación en las Organizaciones & 1 & 0,41 \\
\hline Máster en Dirección de Comunicación Corporativa & 1 & 0,41 \\
\hline Máster en Dirección de Comunicación Empresarial e Institucional & 1 & 0,41 \\
\hline Máster en Comunicación Corporativa e Institucional & 1 & 0,41 \\
\hline Máster en Comunicación Integral & 1 & 0,41 \\
\hline Máster en Comunicación Empresarial e Institucional & 1 & 0,41 \\
\hline Máster en Comunicación Institucional & 1 & 0,41 \\
\hline Máster en Comunicación Corporativa e Institucional Digital & 1 & 0,41 \\
\hline Máster en Publicidad y Comunicación Corporativa e Institucional & 1 & 0,41 \\
\hline Máster en Dirección de Comunicación y Nuevas Tecnologías & 1 & 0,41 \\
\hline Máster Oficial en Gestión Estratégica e Innovación en Comunicación & 1 & 0,41 \\
\hline
\end{tabular}




\begin{tabular}{|c|c|c|}
\hline Máster en Investigación en Comunicación & 1 & 0,41 \\
\hline Máster en Sociología de la Comunicación de Masas & 1 & 0,41 \\
\hline Máster en Comunicación Web 2.0 y Medios Sociales & 1 & 0,41 \\
\hline $\begin{array}{l}\text { Máster en Marketing Electrónico y Nuevas Tecnologías aplicadas a la Gestión y Fidelización de } \\
\text { Clientes a través de la Red }\end{array}$ & 1 & 0,41 \\
\hline Máster en Información Socioeconómica & 1 & 0,41 \\
\hline Máster Europeo en Organización de Congresos y Eventos & 1 & 0,41 \\
\hline Máster en Protocolo, Relaciones Institucionales y Gestión Estratégica de los Eventos & 1 & 0,41 \\
\hline Máster en Protocolo y Organización de Eventos & 1 & 0,41 \\
\hline Máster en Protocolo y Relaciones Institucionales & 1 & 0,41 \\
\hline Máster en Ceremonial y Protocolo & 1 & 0,41 \\
\hline Máster en Comunicación y Protocolo & 1 & 0,41 \\
\hline Máster en Protocolo Oficial y Organización de Eventos & 1 & 0,41 \\
\hline Máster en Comunicación y Marketing & 1 & 0,41 \\
\hline Master en Dirección de Comunicación y Marketing & 1 & 0,41 \\
\hline Máster en Dirección de Marketing y Comunicación & 1 & 0,41 \\
\hline Máster en Marketing y Comunicación Corporativa & 1 & 0,41 \\
\hline Máster en Dirección y Gestión de la Comunicación, Marketing y Publicidad & 1 & 0,41 \\
\hline Máster en Business Communication y Planificación Estratégica de Marketing & 1 & 0,41 \\
\hline Máster en Comunicación en Salud 2.0 & 1 & 0,41 \\
\hline Máster en Marketing Digital \& Inbound Marketing & 1 & 0,41 \\
\hline Master en Marketing Profesional & 1 & 0,41 \\
\hline Máster en Marketing y Comercial & 1 & 0,41 \\
\hline Máster Europeo en Marketing y Negocio Online & 1 & 0,41 \\
\hline Master in Online Marketing and Communications & 1 & 0,41 \\
\hline Máster en Medios de Comunicación & 1 & 0,41 \\
\hline Máster en Social Media Management & 1 & 0,41 \\
\hline Máster en Community Management: Empresa 2.0 y Redes Sociales & 1 & 0,41 \\
\hline Máster en Digital Business & 1 & 0,41 \\
\hline Máster en Estrategia en Redes Sociales & 1 & 0,41 \\
\hline Máster en Comunicación Multimedia & 1 & 0,41 \\
\hline Máster en Gestión y Mantenimiento de Empresas Multimedia & 1 & 0,41 \\
\hline Máster en Ingeniería de Sistemas Microsoft & 1 & 0,41 \\
\hline
\end{tabular}

\begin{tabular}{|c|c|c|}
\hline Máster en Comunicación, Public Affairs, Marketing y Marca & 1 & 0,41 \\
\hline Máster de Experto en Comunicación y Arte & 1 & 0,41 \\
\hline Máster en Dirección en Ventas y Marketing & 1 & 0,41 \\
\hline Máster en Marketing, Dirección de Ventas y Publicidad & 1 & 0,41 \\
\hline Máster en Comunicación Política e Institucional & 1 & 0,41 \\
\hline Máster en Comunicación Política, Institucional y Empresarial & 1 & 0,41 \\
\hline Máster Universitario en Análisis Político & 1 & 0,41 \\
\hline Máster en Comunicación de las Instituciones Públicas y Políticas & 1 & 0,41 \\
\hline Master en Radio & 1 & 0,41 \\
\hline Máster diario «El País» de Periodismo & 1 & 0,41 \\
\hline Máster en Gestión del Conocimiento & 1 & 0,41 \\
\hline Máster Ejecutivo Online en Community Management y Dirección de Redes Sociales & 1 & 0,41 \\
\hline Máster en Dirección y Gestión de Empresas Audiovisuales & 1 & 0,41 \\
\hline Máster en Dirección de la Empresa Audiovisual & 1 & 0,41 \\
\hline Máster en Periodismo y Comunicación Digital & 1 & 0,41 \\
\hline Máster en Periodismo Digital y Comunicación Corporativa & 1 & 0,41 \\
\hline Máster en Comunicación y Periodismo Económico & 1 & 0,41 \\
\hline Máster en Comunicación Periodística, Institucional y Empresarial & 1 & 0,41 \\
\hline Máster en Comunicación Audiovisual y Periodismo & 1 & 0,41 \\
\hline Máster en Publicidad y Comunicación Institucional & 1 & 0,41 \\
\hline Máster en Comunicación y Gestión Política & 1 & 0,41 \\
\hline Máster en Gestión y Dirección Comercial & 1 & 0,41 \\
\hline Máster en Gestión Comercial y de Marketing & 1 & 0,41 \\
\hline Executive Máster en Marketing \& Ventas & 1 & 0,41 \\
\hline Máster en Desarrollo Directivo & 1 & 0,41 \\
\hline Máster en Gestión de Empresas & 1 & 0,41 \\
\hline Máster en Recursos Humanos y Cambio Organizacional & 1 & 0,41 \\
\hline Máster en Dirección de Recursos Humanos & 1 & 0,41 \\
\hline Máster en Recursos Humanos & 1 & 0,41 \\
\hline Máster en Gestión de Recursos Humanos & 1 & 0,41 \\
\hline Máster en Relaciones Laborales & 1 & 0,41 \\
\hline Máster en Liderazgo & 1 & 0,41 \\
\hline
\end{tabular}




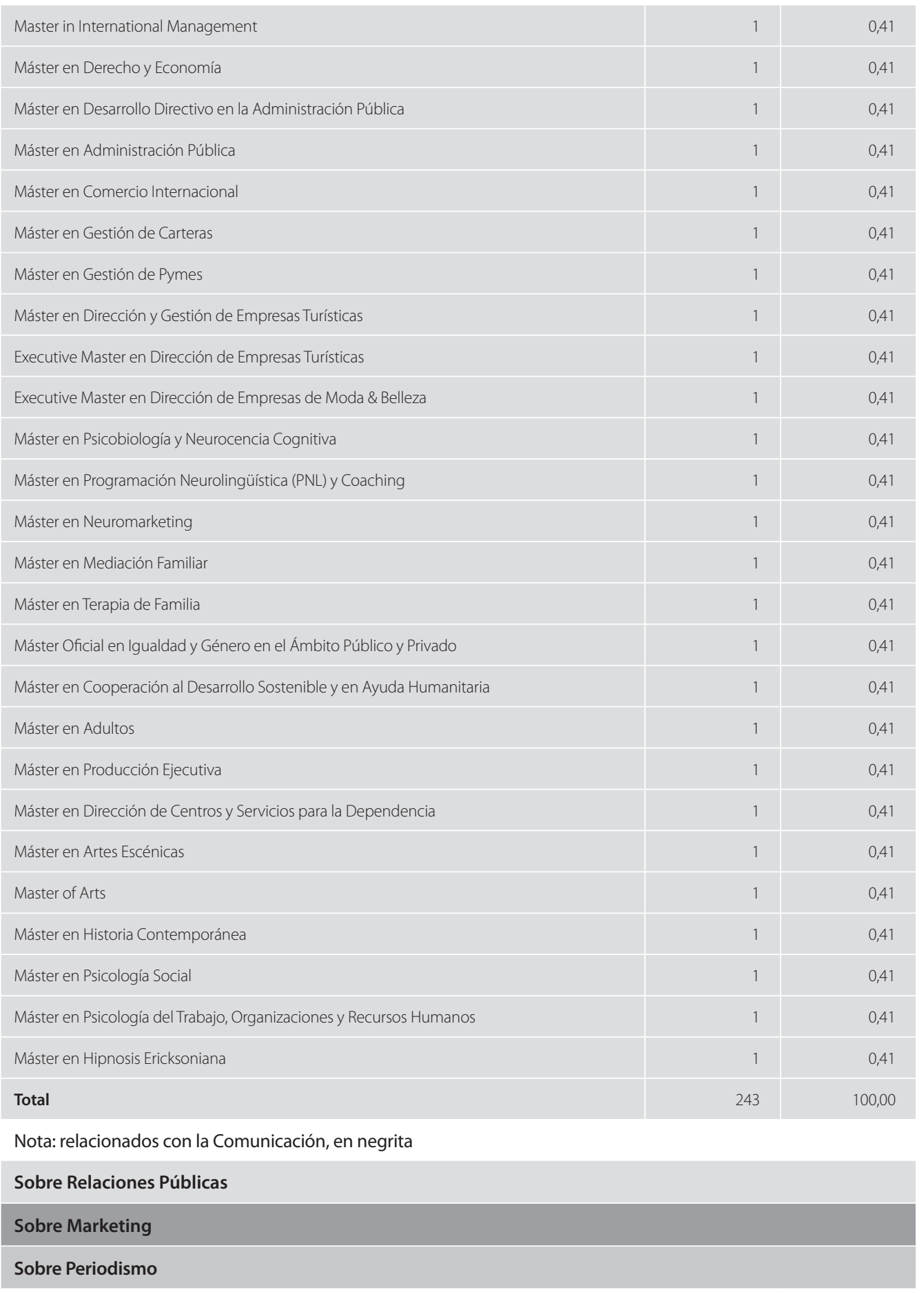

Fuente: Elaboración propia
Fueron 243 (88,68\%) los sujetos que cursa- $\quad$ ter en Periodismo (8 - 3,29\%); y el Máster en ron estudios de máster y/o de master executive, Comunicación Empresarial (6-2,47\%).

de tipologías muy variadas y altamente atomiza- De los 137 másteres, los relacionados con la das (137). Comunicación (en negrita en la Tabla 1) son 83

La formación prevalente es el Master Executi- （60,58\%). Se identifican 3 (2,19\%) que incluyen ve in Business Administation-MBA (44 - 18,11\%), el término «Relaciones Públicas» en su denomiseguida muy de lejos por el Máster en Dirección nación; 11 (8,03\%) el de «Periodismo» o afines Comercial y de Marketing (10 - 4,12\%); el Más- y 22 (16,05\%) el de «Marketing».

\section{Tabla $2 \cdot$ Postgrados}

\begin{tabular}{|l|c|c|}
\hline \multicolumn{1}{|c|}{$N=243$} & $n$ \\
\hline Posgrado en Dirección de Asuntos Públicos & 7 & 10,77 \\
\hline Posgrado en Dirección de Marketing & 3 & 4,62 \\
\hline Posgrado en Periodismo & 3 & 4,62 \\
\hline Posgrado en Dirección de Empresas & 2 & 3,08 \\
\hline Posgrado en Alta Dirección en Gestión Empresarial y Dirección de Comunicación & 2 & 3,08 \\
\hline Posgrado en Comunicación Empresarial & 2 & 3,08 \\
\hline Posgrado en Dirección de Comunicación & 1 & 1,54 \\
\hline Posgrado en Gestión y Dirección de Comunicación & 1 & 1,54 \\
\hline Posgrado en Comunicación Empresarial e Institucional & 1 & 1,54 \\
\hline Posgrado en Dirección Estraté́gica de la Comunicación & 1 & 1,54 \\
\hline Posgrado en Public Affairs and Lobby & 1 & 1,54 \\
\hline Posgrado en Asuntos Públicos & 1 & 1,54 \\
\hline Posgrado en Relaciones Públicas & 1 & 1,54 \\
\hline $\begin{array}{l}\text { Posgrado en Innovaciones en las Relaciones Públicas y Comunicación e Imagen Cor- } \\
\text { porativas }\end{array}$ & 1 & 1,54 \\
\hline Posgrado en Relaciones Institucionales y Protocolo & 1 & 1,54 \\
\hline Posgrado en Dirección de Responsabilidad Corporativa & 1,54 \\
\hline Posgrado Iberoamericano de Responsabilidad Social & 1 & 1,54 \\
\hline Posgrado en Comunicación Interna Aplicada a la Gestión de Personas & 1,54 \\
\hline Posgrado en Community Manager & 1,54 \\
\hline Posgrado en Gestión de la Innovación y el Conocimiento de las Organizaciones & 1,54 \\
\hline Posgrado en Marketing y Comunicación Corporativa & 1,54 \\
\hline Posgrado OBS en Marketing Online & 1 & 1,54 \\
\hline & 1 & 1 \\
\hline
\end{tabular}




\begin{tabular}{|l|l|l|}
\hline Posgrado en Marketing & 1 & 1,54 \\
\hline Posgrado en Marketing y Marketing Digital & 1 & 1,54 \\
\hline Posgrado en TV, Radio and Electronic Journalism & 1 & 1,54 \\
\hline Posgrado de Especialista en Gabinete de Prensa en Empresas e Instituciones & 1 & 1,54 \\
\hline Posgrado en Información Periodistica y Deporte & 1 & 1,54 \\
\hline Posgrado en Información Económica & 1 & 1,54 \\
\hline Posgrado en Periodismo Turístico & 1 & 1,54 \\
\hline Posgrado en Fotoperiodismo & 1 & 1,54 \\
\hline Postgraduate Certificate in Journalism, Web-based Journalism and Online Production & 1 & 1,54 \\
\hline Posgrado en Comunicación Política e Institucional & 1 & 1,54 \\
\hline Posgrado en Comunicación Política y Democracia & 1,54 \\
\hline Posgrado en Political Management & 1 & 1,54 \\
\hline Posgrado en Sociolingǘstica y Comunicación & 1 & 1,54 \\
\hline Posgrado en Derecho Internacional & 1 & 1,54 \\
\hline Posgrado en Comunidades Europeas & 1 & 1,54 \\
\hline Posgrado en Cooperación Internacional & 1 & 1,54 \\
\hline Posgrado en Economía y Administración de Empresas & 1,54 \\
\hline Posgrado en Asesoría Fiscal de Empresas & 1 & 1,54 \\
\hline Posgrado OBS en Marketing Online & 1 & 1,54 \\
\hline Posgraduate Diploma in Management Studies & 1,54 \\
\hline Posgrado en Dirección ONG & 1 & 1,54 \\
\hline Posgrado en Dirección de Fundaciones & 1,54 \\
\hline Posgrado en Administración de Empresas & 1 & 1,54 \\
\hline Posgrado en Comercio Exterior & 1,54 \\
\hline Posgrado en Gestión Cultural & 1,54 \\
\hline Posgrado en Relaciones Laborales & 1 & 1,54 \\
\hline Posgrado en Informática & 1 & 1 \\
\hline Posgrado en Teoría Crritica del Teatro & 1 & 1 \\
\hline Total & 1 & 1 \\
\hline & 1 & 1 \\
\hline
\end{tabular}

Nota: relacionados con la Comunicación, en negrita

\section{Sobre Relaciones Públicas}

Sobre Marketing

Sobre Periodismo

Fuente: Elaboración propia
De los 274 dircoms, 65 (23,72\%) poseen estu- Contemplan el término «Relaciones Públicas» dios de postgrado. Se identifican 49 tipos distin- únicamente $2(4,08 \%, n=49)$, por debajo de los tos de postgrados cursados, de los cuales en tor- $6(12,24 \%, n=49)$ que incluyen el concepto no a la mitad (25-51,02\%) están relacionados «Marketing»; y de los $8(16,32 \%, n=49)$ con el con la Comunicación (en negrita en la tabla 2). concepto «Periodismo» o afines.

\section{Tabla 3 Programas}

\begin{tabular}{|c|c|c|}
\hline $\begin{array}{l}\text { Programa, Programa Executive y Programa Superior } \\
\qquad \mathrm{N}=91\end{array}$ & $\mathrm{n}$ & $\%$ \\
\hline Programa de Desarrollo Directivo (PDD) & 17 & 18,68 \\
\hline Programa Dirección General (PDG) & 12 & 13,19 \\
\hline Programa Superior de Gestión Empresarial y Dirección de Comunicación & 8 & 8,79 \\
\hline Programa de Alta Dirección de Empresas (PADE) & 8 & 8,79 \\
\hline Program for Management Development (PMD) & 5 & 5,49 \\
\hline Programa Superior en Gestión Empresarial y Dirección de Comunicación & 4 & 4,40 \\
\hline Programa Superior de Mujer y Liderazgo & 2 & 2,20 \\
\hline Advanced Management Program & 2 & 2,20 \\
\hline Programa Superior de Responsabilidad Corporativa & 2 & 2,20 \\
\hline Programa Superior de Gestión Empresarial & 2 & 2,20 \\
\hline Programa Executive en Dirección de Comunicación & 1 & 1,10 \\
\hline Programa Superior en Comunicación y Relaciones Públicas & 1 & 1,10 \\
\hline Programa de Dirección Estratégica de la Comunicación Corporativa y de Marketing & 1 & 1,10 \\
\hline Programa Superior en Dirección de Empresas y Comunicación & 1 & 1,10 \\
\hline Executive Education Dirección Comunicación Empresas & 1 & 1,10 \\
\hline Programa de Formación en Gestión de la Reputación Corporativa & 1 & 1,10 \\
\hline Programa Superior en RC-Reputación Corporativa & 1 & 1,10 \\
\hline Programa Ejecutivo en Reputación Corporativa & 1 & 1,10 \\
\hline Programa Ejecutivo en RSC y Sostenibilidad & 1 & 1,10 \\
\hline Programa Superior de Lobby & 1 & 1,10 \\
\hline Corporate Diplomacy \& Public Affairs Executive Program & 1 & 1,10 \\
\hline Programa Estrategias de Patrocinio Deportivo y Mecenazgo Cultural & 1 & 1,10 \\
\hline Programa Internacional de Comunicación Política y Gobierno en la América del s. XXI & 1 & 1,10 \\
\hline Programa Superior en Comunicación y Gestión Empresarial & 1 & 1,10 \\
\hline
\end{tabular}




\begin{tabular}{|l|c|c|}
\hline Programa Integral de Management & 1 & 1,10 \\
\hline Advanced Strategic Management Program & 1 & 1,10 \\
\hline Programa Superior en Gestión de Empresas (PSGE) & 1 & 1,10 \\
\hline Programa Superior de Liderazgo & 1 & 1,10 \\
\hline Programa Gestión Directiva & 1 & 1,10 \\
\hline Programa Superior en Internet Business & 1 & 1,10 \\
\hline Programa Superior en Dirección de Fundaciones & 1 & 1,10 \\
\hline Executive Education & 1 & 1,10 \\
\hline Programa Superior de Estudios Avanzados de la Edición & 1 & 1,10 \\
\hline Programa Superior en Marketing Operativo (PSMO) & 1 & 1,10 \\
\hline Programa de Dirección de Marketing Digital & 1 & 1,10 \\
\hline Program for Marketing Management (PMM) & 1 & 1,10 \\
\hline Programa en Desarrollo Comercial y Marketing & 1 & 1,10 \\
\hline Digital Business Executive Program (DIBEX) & 1 & 1,10 \\
\hline Value Innovation Program & 1 & 1,10 \\
\hline Total & 91 & 100,00 \\
\hline
\end{tabular}

Nota: relacionados con la Comunicación, en negrita

\section{Sobre Relaciones Públicas}

\section{Sobre Marketing}

Sobre Periodismo

Fuente: Elaboración propia

Los dircoms que han realizado estudios tipo programa, programa executive y/o programa superior son 91 (33,21\%). Se identifican 38 tipos distintos de programas. Los relacionados con la Comunicación son 15 (39,47\%, n=38) (en negrita en la Tabla 3)

Únicamente 1 (2,63\%, n=38) ostenta el término «Relaciones Públicas» en su nomenclatura; $5(13,15 \%, n=38)$ el de «Marketing», no habiendo ninguno $(0 \%, \mathrm{n}=38)$ relacionado con el «Periodismo» o afines.

Lidera el ranking un Programa de Desarrollo Directivo-PDD, cursado por 17 (18,68\%, n=91) dircoms, seguido de un Programa de Dirección General-PDG (12 - 13,19\%, n=91) y, empatados, un Programa de Alta Dirección de Empresas-PADE y un Programa Superior de Gestión Empresarial y Dirección de Comunicación, con 8 dircoms $(8,79 \%, \mathrm{n}=91)$, respectivamente, entre sus alumni.

\section{Conclusiones y Discusión}

Los resultados del estudio proporcionan respuesta a las tres preguntas de investigación: de la muestra de dircoms españoles analizados, 243 $(88,68 \%, n=274)$ han cursado estudios de más- ter y/o de master executive (PI1); 65 (23,72\%, $\mathrm{n}=274)$ de posgrado (PI2) y 91 (33,21\%, n=274) de programa, programa executive y/o programa superior (PI3), identificándose la notable atomización y dispersión de las formaciones cursadas, respectivamente, en las Tablas 1, 2 y 3.

En la formación de máster es mayoritaria la de tipo MBA $(44-18,11 \%, \mathrm{n}=137)$ y la relacionada con la Comunicación es prevalente (83 $60,58 \%, n=137$ ), seguida muy de lejos por las de Marketing ( $22-16,05 \%, n=137$ ), Periodismo $(11-8,03 \%, n=137)$ y Relaciones Públicas $(3-2,19 \%, n=137)$

Entre los posgrados cursados, poco más de la mitad están relacionados con la Comunicación ( $25-51,02 \%, n=49)$, seguidos por los especializados en Periodismo ( $8-16,32 \%, \mathrm{n}=49)$, Marketing $(6-12,24, \mathrm{n}=49)$ y RP $(2-4,08 \%, \mathrm{n}=49)$.

Los estudios tipo programa están liderados por los especializados en Management: PDD (17 - 18,68\%, n=91); PDG (12 - 13,19\%, n= 91); y PADE $(8-8,79 \%, n=91)$ y los relacionados con Comunicación son prevalentes (15 - 39,47\%, $\mathrm{n}=38$ ), seguidos por los de Marketing (5 13,15\%, n=38), y Relaciones Públicas (1 2,63\%, n=38). El Periodismo no ha sido elegido como opción de especialización por ninguno de los dircoms de la muestra.

Los datos de las respuestas obtenidas permiten verificar y/o refutar las cinco hipótesis planteadas inicialmente:

H1 Se verifica parcialmente la $1^{\mathrm{a}}$ hipótesis, ya que, si bien los programas formativos especializados de forma genérica en Comunicación son mayoritarios en las tres tipologías analizadas, los dircoms que han elegido un MBA entre los másteres y un PDD, un PDG o un PADE entre los programas alcanzan la supremacía.
H2 Se refuta la $2^{\text {a }}$ hipótesis, ya que en las tres tipologías de estudios analizadas la formación en Relaciones Públicas queda relegada a posiciones minoritarias

H3 Se refuta la $3^{a}$ hipótesis, ya que en ninguna de las tipologías de estudios analizadas logra el liderazgo el Periodismo, y si bien ocupa la segunda posición entre los posgrados, es total la carencia de elección de esta opción de especialización entre los programas.

H4 Se refuta la $4^{a}$ hipótesis, si bien se observa un interés medio por adquirir conocimientos en Marketing en las tres tipologías de estudios analizadas.

H5 Se verifica parcialmente la $5^{a}$ hipótesis, ya que es el Management la opción mayoritaria en másteres y programas, aunque no en posgrados.

La gran oferta española de formación de postgrado especializada en Comunicación y Relaciones Públicas (Hernández et al., 2009; Almansa y Athaydes, 2012; García y Gómez, 2012a, 2012b; Castillo et al., 2013; Matilla et al., 2014) se refrenda con los datos obtenidos sobre la fuerte atomización y alta dispersión en las tres tipologías de variables analizadas.

En congruencia con estudios homólogos europeos (ECM, 2015: 14), se desprende que buena parte de los dircoms españoles confían la construcción de sus carreras a la adquisición de conocimientos académicos (ECM, 2012, p. 77) y optan por especializarse mediante formación de postgrado, en consistencia con otros estudios similares realizados anteriormente en España (Dircom, 2005, 2010, 2015b, 2018) que, en el caso de los másteres y de los programas analizados se orienta claramente a la gestión (Management), lo cual resulta consistente con Dircom (2013), ya que ello permite a los dircoms más seniors integrarse en condiciones óptimas en el 
comité de dirección, entender cómo alinear adecuadamente el plan estratégico de comunicación con el corporativo y de negocio y asesorar (staff) a sus compañeros funcionales $\mathrm{y}$ a sus superiores jerárquicos en materia comunicativa, con la finalizar de homogeneizar los mensajes emitidos a sus públicos y stakeholders.

Los postgrados que, en sus más variadas y múltiples formas, permiten especializarse en Comunicación son los elegidos mayoritariamente por los sujetos de la muestra en las tres tipologías de variables analizadas, colocando en posiciones intermedias la especialización en Marketing, en lugares inferiores al Periodismo y totalmente marginales a las Relaciones Públicas

La literatura analizada coincide en que la formación de grado (licenciaturas con anterioridad a la implantación del EEES) mayoritaria de los dircoms españoles es el Periodismo, seguida a gran distancia por las Ciencias Empresariales y, minoritariamente, por la Publicidad y las Relaciones Públicas (Dircom, 2005, 2010, 2015b, 2018; García, 2013; prNoticias.com, 2014a).

Podría especularse que la prevalencia de la formación previa de grado en Periodismo proporciona a los dircoms españoles con esa formación los conocimientos auto-considerados suficientes para la praxis «desde el otro lado» y que radique en este hecho que los estudios de postgrado sobre la materia no susciten su interés para especializarse, al contrario de lo que podría ocurrir entre el menor número de los graduados en Ciencias Empresariales u otras disciplinas no relacionadas con la comunicación, quienes sí buscarían nuevos conocimientos sobre Periodismo para su ejercicio profesional en el ámbito comunicativo. García (2013: 223) afirma que la concepción de la profesión y de cómo se ejerce está directamente relacionada con la formación previa recibida y atribuye a la prevalencia de la formación de grado en Periodismo la deficiente profesionalización de los dircoms españoles, quienes desarrollan mayoritariamente su función en torno a la técnica de la Publicity y desde l reduccionismo táctico del simple gabinete de prensa interno, datos congruentes con ADECEC (2008), prNoticias (2014a y 2014b) y Sáez et al. (2012: 77) y muy similares a los estadounidenses, donde la vinculación de los profesionales con la publicity y el media relations es también mayoritaria (Sha, 2011).

Llama la atención que la formación de postgrado en Relaciones Públicas cursada sea tan escasa $(2,19 \%$ en máster; 4,08\% en postgrado; $13,15 \%$ en programas), ya que sus diversas técnicas son las de uso fundamental en un departamento de comunicación (Dircom Cataluña, 2013, p. 14), que todo dircom debería conocer en profundidad para una adecuada praxis de su función y para desempeñarla desde una vertiente más estratégica y menos táctica.

Dado que la formación de grado en Relaciones Públicas se observa que se oferta con graves nomalías y severas carencias en el sistema universitario español (Moreno, 2004; Moreno et al., 2014; Xifra, 2015), no logrando satisfacer las expectativas de los empleadores (Xifra, 2015: 171) y dificultando la contratación de sus egresados, no es de extrañar que la formación de grado en Relaciones Públicas recibida por los dircoms analizados se sitúe en posiciones marginales, en consistencia con diversos estudios anteriores realizados por Dircom: 5,6\% en 2005 y 5,3\% en 2010 (Dircom, 2005, 2010).

Todo parece apuntar, pues, a que la formación de postgrado en Relaciones Públicas no es percibida como una segunda oportunidad de mejora de dichos conocimientos académicos de grado entre los dircoms españoles (Matilla et al., 2014, p. 371; Dircom, 2018), probablemente por des- conocimiento de lo que realmente son y de su alcance total o por el descrédito del término sufrido los últimos años, que ha sido sustituido por otras denominaciones, tanto en España como en otros países europeos (Moreno et al., 2012). Los gaps existentes entre la oferta formativa universitaria en Relaciones Públicas y las necesidades del mercado laboral de acogida (Miquel Segarra 2018) no son patrimonio únicamente español, ya que la situación es muy parecida en Norteamérica (Soloski, 1994; Aldoory y Toth, 2000 Hon et al., 2004; Wright y Turk, 2007).

En Latinoamérica casi el 40\% (39,7\%) de los dircoms participantes en el «LCM 2015» contaban con un grado en su curriculum académico, frente al 51,8\% que había cursado un máster y un 7,5\% que había alcanzado doctorarse, por lo que su situación es similar a la española, la europea y la estadounidense en lo que respecta al acceso a la profesión mediante estudios superiores, si bien se observan variaciones notables entre países.

A diferencia del panorama español y estadounidense, en varios de los países latinoamericanos analizados en la literatura consultada los estudios universitarios sobre Relaciones Públicas son de creación reciente, por lo que la mayoría de los profesionales en ejercicio son egresados en Comunicación Social, echando en falta una formación especializada en Relaciones Públicas con una orientación estratégica para una mejora en la amplitud y calidad de su praxis.

También es consistente con los contextos español y europeo descritos en la literatura que pese a la gran expansión sufrida por la profesión en América Latina, se identifica claramente la falta de conocimientos de empresarios, CEOs y directivos de empresas y organizaciones e, incluso, de los propios dircoms, sobre las funciones que deben desarrollarse en un departamento de comunicación, confundiéndose a menudo las actividades de Relaciones Públicas con la simple organización de eventos (Moreno y Molleda 2006), por lo que se mantienen en su dimensión más técnica -táctica- (Ordeix y Rom, 2014, p 51) y, en general, echándose en falta una orientación estratégica (Moreno et al., 2006).

La concentración de las funciones del departamento de comunicación en torno al media relations es prevalente en la mayoría de los países de cono Sur analizados en la literatura, destacando que las relaciones con la prensa experimentarán un incremento en los tres años siguientes (LCM 2015), por lo que, de cumplirse la previsión, la actualmente deficiente profesionalización tenderá a perpetuarse como está hoy y a no evolucionar a mejor

En Colombia los dircoms se quejan del intrusismo profesional practicado por los periodistas y también destacan que los altos directivos empresariales «no comprenden el valor de un proceso sistemático de comunicación como una estrategia fundamental empresarial» (Moreno et al., 2006. P. n.e.)

En general, en América del Sur y en Centroamérica la literatura muestra que no se distinguen adecuadamente las diferencias entre la comunicación corporativa, el marketing, la publicidad o el periodismo (Argentina 47,5\%; Brasil 54,7\% Chile 41,8\%; Colombia 39,6\%; Costa Rica 47,2\%; México 48,5\%; Perú 55,6\%; Rep. Dominicana 38,3\%; Venezuela 41,9\%) (LCM, 2015), por lo que podría especularse que la oferta formativa existente en el cono Sur especializada en relaciones públicas y en comunicación corporativa, a menudo de reciente creación en varios países, no contribuye a clarificar el confusionismo reinante en el área geográfica analizada, en consistencia con las realidades española, europea y norteamericana, donde es mayoritaria la concepción táctica de la gestión profesional de la comu- 
nicación como sinónimo prevalente de mero gabinete de prensa, pese a que este tipo de estudios cuentan con un histórico de varias décadas.

Todo parece apuntar, como tendencia, que aún queda mucho camino por recorrer para que los grados y postgrados cursados por los dircoms de los dos continentes analizados y la concepción unitaria y completa de la función entre empresas y profesionales converjan, de forma sistémica, en un punto de confluencia común que se adecue al perfil funcional holístico, integrado e integral de la función comunicativa postulado por Dircom (2013) desde la orientación propugnada por la comunicación corporativa y que emplee los conocimientos especializados en relaciones públicas descritos por Dircom Cataluña (2013).

Mientras perdure la carencia de criterios comunes de todos los actores implicados, la posición podría seguir nutriéndose de profesionales formados a nivel de postgrado en muy diversas y atomizadas áreas de conocimiento y, así, los directores de recursos humanos de ambos lados del Atlántico tardarán en disponer de un criterio adecuado, común y ampliamente compartido sobre cuál debe ser el perfil óptimo de conocimientos y competencias adquiridos mediante formación de postgrado universitario y requerido para un ejercicio de calidad de la función, sobre todo en lo que a su orientación estratégica se refiere.

\section{Limitaciones}

Como limitación al estudio se destaca que en el directorio analizado únicamente figura algo menos de la mitad $(46,13 \%)$ del universo total de los socios de Dircom que han autorizado la difusión pública de sus datos personales en dicha publicación.

Otra limitación consiste en que no todos los profesionales españoles de las relaciones públicas y de la comunicación corporativa en activo que configuran el universo total están asociados a Dircom, constituyendo la muestra de 274 dircoms un porcentaje pequeño que no permite realizar un mapa generalizado y conclusivo del objeto de estudio, aunque puede considerarse una muestra de oportunidad altamente representativa del universo total de este tipo de profesionales españoles y, así, los resultados obtenidos permiten ser considerados como tendencias.

\section{Futuras líneas de investigación} y extensiones

Los resultados obtenidos aconsejan mantener abierta esta línea de investigación sobre la vinculación entre estudios y praxis de los dircoms españoles, para verificar periódicamente si, en los próximos años, se producen alteraciones significativas en el objeto de estudio y, pese a sus limitaciones, poder proporcionar información sobre nuevas tendencias de posible utilidad para los directores de recursos humanos -y para el propio colectivo profesional-, responsables de definir los criterios de contratación en cuanto al perfil de formación de los dircoms y de tipología de formación continua óptima, animando la ampliación de este trabajo con nuevos análisis comparativos internacionales y procurando, con ello, evitar favorecer el intrusismo con contrataciones amparadas en curricula formativos inadecuados.

Como extensión, los resultados también sugieren la conveniencia de realizar estudios sobre la formación de grado cursada por la misma muestra analizada para contribuir a cartografiar un mapa formativo universitario de referencia más rico y completo, ya que los estudios de postgrado españoles suelen cursarse para obtener una especialización profesional tras la consecución de un grado, por lo que se trataría de formaciones complementarias que deberían analizarse conjunta y periódicamente los próximos años.

\section{Bibliografía}

ADECEC (2008). La comunicación y las relaciones públicas en España. Radiografia de un sector. Madrid· ADECEC.

Almansa, A. y Athaydes, A. (2012). Los posgrados en Relaciones Públicas en las universidades españolas. Estudio sobre su situación y temáticas. En E. Ordeix y J. Rom (eds.), La innovación en Relaciones Püblicas (pp. 315-330). Barcelona: Trípodos (número especial). Araujo de la Mata, A, Barrutia Güenaga J, Hoyos Iruarrizaga, J., Rodríguez, J. e Ibáñez Hernández, P. (2006). Comportamiento de las empresas respecto a la formación continua de sus directivos. Cuadernos de Gestión, 6(1), 83-98.

Castillo Esparcia, A., Álvarez Nobell, A. y Muñiz Velázquez, J.A. (2013). EEES y la formación en comunicación en España. La estructura de la oferta de posgrado. Estudios sobre el Mensaje Periodístico, 19, número especial, marzo, 99110.

CSIC-Centro Superior de Investigaciones Cientificas. Ranking SPI - Áreas de conocimiento adscritas a cada disciplina. http://epuc.cchs.csic.es/ SPI/AC_AT.pdf [Último acceso 21/02/2018

Dircom, 2005. El estado de la comunicación en España. Madrid.

Dircom, 2010. El estado de la comunicación en España. Madrid Dircom.

Dircom, 2013. Manual de la Comunicación. Madrid: Dircom. Dircom, 2015a. Quién es quién en Dircom. Madrid: Dircom. Dircom, 2015b. El estado de la comunicación en España. Madrid: Dircom.

Dircom, 2018. Anuario de la Comunicación 2018. Madrid. Dircom.

Dircom Catalunya (2013). Decálogo dircom. 10 preguntas y 10 respuestas sobre la función de Dirección de Comunicación Barcelona: Dircom Cataluña. http://www.dircom.org/catalunya/decalogo-dircom.pdf [Último acceso 21/02/2018]. ECM-European Communication Monitor (2012). Challenges and competencies for strategic communication. Results of a Survey in 42 Countries. Brussels: EACD/EUPRERA, Helios Media. http://de.slideshare.net/communicationmonitor/european-communication-monitor-ecm-2012-results-chartversion [Último acceso 21/02/2018].

ECM-European Communication Monitor (2015). Creating communications value through listening messaging and measu- rement. Results of a Survey in 41 Countries. Brussels: EACD/ EUPRERA, Helios Media. http://www.zerfass.de/ECM-WEBSITE/media/ECM2015-Results-ChartVersion.pdf [Último acceso 21/02/2018].

García, C. (2013). Clientelism, Economic Structure and Public Relations in Southern Europe: An Exemple of Diversity in the Western World. Public Relations Journal, 7(2), 214-241

García Jiménez, A. y Gómez Mompart, J.L. (2012a). Posgrado en Comunicación: una primera aproximación a la situación en España. En M.I. Vasallo de Lopes, Posgrados en Comunicación en Iberoamérica. Politicas Nacionales e Internacionales (pp. 81-111). São Paulo, Brasil: ECAUSP

García Jiménez, A. y Gómez Mompart, J.L., (2012b). Posgrado en Comunicación: una primera aproximación a la situación en España. En M. K. Kunsch, J. Marqués de Melo, Comunicação Ibero-americana. Sistemas Mediáticos, Diversidade Cultural, Pesquisa e PósGraduação (pp. 261-278). São Paulo, Brasil: SOCICOM, CONFIBERCOM.

Hernández, S., Losada, J.C. y Matilla, K. (2009). Las Relaciones Públicas y la Comunicación Corporativa en la oferta universitaria de postgrado del estado español: análisis sistemático y proyectivo y una iniciativa de observatorio del estado de la situación por parte de una asociación profesional. Razón y Palabra, 14(70), número especial «Relaciones Públicas», 1-18.

Hon, L.C., Fitzpatrick, K.R. y Hall, M.R. (2004). Searching for 'the Ideal' Graduate Public Relations Curriculum. Journalism and Mass Communication Educator, 59(2), 125-142.

JEL Classification System» (Journal of Economic Literature). http://www.aeaweb.org/econlitjelCodes.php?view=je [Último acceso 16/02/2018].

Krippendorff, K. (2004). Content Analysis: An Introduction to its Methodology. Thousand Oaks, CA: Sage ( $2^{\mathrm{a}} \mathrm{ed}$.

Landeta, J., Barrutia, J., Hoyos, J. y Araujo, A. (2015). Initiatives for the improvement of continuous management training. Cuadernos de Gestión, 15(1), 61-92

LCM-Latin-American Communication Monitor (2015). Latin-American Communication Monitor (LCM) 2014-2015. Excelencia en comunicación estratégica, trabajo en la era digital 'social media' y profesionalización. Resultados de una encuesta en 18 paises. Bruselas: EUPRERA y Dircom. http://wwwlatincom.info/que-es-el-lcm/ [Último acceso 21/02/2018].

Marzo Navarro, M., Pedraja Iglesias, M. y Rivera Torres, P 


\section{4 aDResearch ESIC}

fior: El caso de las Ingenierías. Cuadernos de Gestión, 6(1), 27-44.

Matilla, K., Cuenca Fontbona, J. y Marca Francés, G. 2014). Ya me he graduado en Relaciones Públicas. Y, ahora, zqué? Una mirada a la formación de postgrado oficial e España (2013-2014). En J. Fombona Cadavieco y D. Cald villa Domínguez, Nuevas formulaciones de los contenidos docentes (pp. 371-384, capitulo 32). Madrid: McGraw Hill Education.

Ministerio de Educación, Cultura y Deporte (2016). Datos y cifras del sistema universitario español. Curso 2015-2016. http://www.mecd.gob.es/educacion-mecd/areas-educacion/ universidades/estadisticas-informes/datos-cifras.html [Último acceso 21/02/2018]

Miquel Segarra, S.; López Font, L. y Gil Soldevila, S. (2018) Radiografia de las consultoras de comunicación en Españ perfil profesional, estructura y actividad. Revista Latina de Comunicación Social, 73, 478-503.

Miquel-Segarra, S., (2016). Análisis de los profesionales de a comunicación corporativa desde el asociacionismo en España. Business, Economy and Society: Issues for Research in Knowledge and Information Society. https://rua.ua.es/dspace/ bitstream/10045/69561/1/tesis_susana_miquel_segarra. pdf, (20 de septiembre de 2018).

Molleda, J.C., Moreno, A., Athaydes, A. y Suárez, A.M (2009). Macroencuesta latinoamericana de comunicación relaciones públicas. Organicom, Revista Brasileira de Comuncação Organizacional e Relaçoes Públicas, 7(13), 118-141

Moreno-Fernández, A., Carrasco-Campos, A. y Saperas-La piedra, E. (2014). Los efectos del proceso Bolonia en el curículum de las Relaciones Públicas. Análisis del estado de cuestión. Sphera Pública, junio, número especial, 163-184 Moreno-Fernández, M.A., Navarro-Ruiz, C. y Zerfass, A (2012). Relaciones Públicas, un término desacreditado en España y el resto de Europa. Conclusiones del ECM 2011. Hologramática - Facultad de Ciencias Sociales UNLZ, 17(2), 115-140.

Moreno, A., Molleda, J.C. y Suárez, A.M. (2006). Comunicación estratégica y relaciones públicas en entornos socio conómicos y políticos en transición: estudio comparativo en Colombia, México y Venezuela. Razón y Palabra, 51.

Moreno, A. y Molleda, J.C. (2006). Las relaciones públicas en México: contextos económico, político y mediático en un proceso histórico de cambios. Razón y Palabra, 48.
Oliveira, I. de L. (2014). Fronteiras entre o campo da Comunicação Organizacional e das Relaçōes Públicas: um perspectiva dos estudos brasileiros. En A. González Herrer y Mª. Huertas Roig, (coords.), La conducta interactiva de los públicos para las relaciones eficaces. Investigación aplicada en la estrategia de Relaciones Públicas (pp. 163-178). Zaragoza, España: Universidad San Jorge, AIRP \& Dircom

Ordeix Rigo, E. y Rom Rodríguez, J. (2014). Estudio crítico comparativo cas entre Chile y España a partir de los principios del estudio de la excelencia. En A. González Herrero y M ${ }^{a}$ A. Huertas Roig (coords.), La conducta interactiva de los públicos para las relaciones eficaces (pp. 41-54). Investigación aplicada en la estrategia de Relaciones Públicas. Zaragoza, España: Universidad San Jorge, AIRP \& Dircom.

prNoticias.com (2014a). 6 de cada 10 directores de comunicación estudiaron periodismo, 10 julio. Disponible en: https:// prnoticias.com/comunicacionpr/541-prcomunicacion-1/20132456-6-de-cada-10-directores-de-comunic cion-estudiaron-periodismo [Último acceso 21/09/2018]

prNoticias.com (2014b). La reputación, el activo estratégico mas valorado dentro de los departamentos de comunicación, 6 octubre. Disponible en: https://prnoticias.com/comunicacionpr/527 barometro-de-prcomunicacion-1/20134507-la-reputacionl-activo-estrategico-mas-valorado-dentro-de-los-departamentos-de-comunicacion [Último acceso 21/09/2018]

Rivera, O., del Orden, O. y Ranguelov, S. (2003). Análisis de la oferta de formación de postgrado en gestión de conocimiento, Cuadernos de Gestión, 3(1), 27-47.

Sáez, A., Matilla, K. y Cuenca, J. (2015). De la comunicació empresarial i institucional a b comunicació corponativa: reptes de futur. En M. Civil Serra et al. (eds.), Informe de la comunicació a Catalunya 2013-2014 (pp. 243-270). Barcelona: Generalitat de Catalunya, Institut de la Comunicació de la Universitat Autònoma de Barcelona (InCom-UAB) Colllecció Lexikon Informes, 4. http://incom.uab.cat/informe/download/2013/informe13_pdf.pdf [Último acceso 21/02/2018]

Sha, B.L. (2011). Practice Analysis: Professional competencies and work categories in public relations today. Public Relations Review, 27, 187-196

Soloski, J. (1994). On defining the nature of graduate education. Journalism Educator, 49, 12-17.

Universidad de Granada (2014). Ranking I-IGR de Universiades Españolas según Campos y Disciplinas Cientificas - edición - Periodo 2009-2013. Grupos de investigación «EC3EC3 Metrics spin-off» y «SCI2S». Granada, España. www rankinguniversidades.es/ [Último acceso 21/02/2018

Van Ruler, B. (2005). Commentary: professionals are from Venus, scholars are from Mars. Public Relations Review, 31 159-173.
Wright, D K. y Turk, JV (2007). Public relations knowledge and professionalism: Challenges to educators and practitioners. En E.L. Toth (ed.), The future of excellence in public relations and communication management: Challenges for the next generation (pp. 571-588). Mahwah, NJ: Lawrence Erlbaum. 